\title{
Amiloidosi, il percorso diagnostico. Quando pensarci e come trovarla
}

\author{
Silvia Farsetti ${ }^{1}$, Anna Maria Ciciani ${ }^{2}$, Giuseppe Curciarello ${ }^{3}$, Egrina Dervishi ${ }^{1}$, Santi Nigrelli ${ }^{4}$, Franco Bergesio ${ }^{5}$ \\ ${ }^{1}$ SOD Nefrologia, Dialisi e Trapianto, Azienda Ospedaliero-Universitaria Careggi, Firenze \\ ${ }^{2}$ SOD Nefrologia e Dialisi, Nuovo Ospedale S. Giovanni di Dio, Firenze \\ ${ }^{3}$ SOD Ematologia Clinica e Oncoematologia, USL Toscana Centro, Firenze \\ ${ }^{4}$ SOD Nefrologia e Dialisi, Ospedale S. Maria Annunziata, Firenze \\ ${ }^{5}$ Centro Fiorentino per lo studio e la cura delle Amiloidosi, Firenze
}

\begin{abstract}
The diagnostic path of amyloidosis. When to think of it and how to find it
The diagnosis of amyloidosis has been difficult and troublesome until the introduction of periombelical fat tissue aspirate as the method of choice for tissue biopsy. Nowadays the diagnostic path includes three main steps: suspicion of the disease, periombelical fat tissue aspirate or biopsy of the involved organ(s), and identification of the amyloidogenic protein. Several clinical and biohumoral conditions are highly suggestive of the disease, such as MGUS or multiple myeloma for AL, chronic inflammatory diseases for AA, and a family history of cardiac or peripheral neuropathy for the hereditary forms. However, in case of a tissue sample positive for Congo red staining, these conditions are not sufficient to identify the type of amyloid fibrils. Indeed misdiagnosis, especially in the presence of MGUS, is very likely and deserves particular attention. In most cases traditional immunohistochemistry is no longer adequate and there is a need for further investigations such as immunoelectron microscopy (immunogold) or proteomics with laser dissection and mass spectroscopy. Chemotherapy cannot be started until a definite diagnosis of the type of amyloidosis has been made.
\end{abstract}

Keywords: Biopsy, Congo red staining, Diagnosis, Fat tissue aspirate, Immunohistochemistry, Proteomics

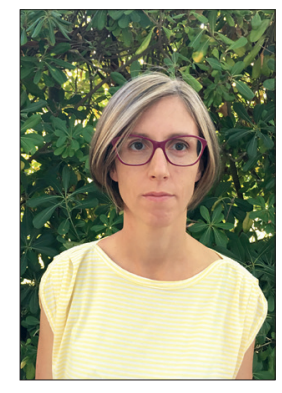

Silvia Farsetti

determinato tessuto. E, se non bastasse, to con rosso Congo) in luce polarizzata tecnica che, ancorché

Accepted: July 6, 2017

Published online: September 13, 2017

Indirizzo per la corrispondenza:

Dr.ssa Silvia Farsetti

SOD Nefrologia, Dialisi e Trapianto

AOUC Azienda Ospedaliero-Universitaria Careggi

Largo Brambilla, 3

50134 Firenze

silvia.farsetti@email.it semplice, richiede tuttavia un minimo di esperienza.

Tutte queste ragioni hanno penalizzato il percorso diagnostico dell'amiloidosi fin tanto che non è stata proposta e si è diffusa la tecnica dell'ago-aspirato del grasso periombelicale, che ha rappresentato una vera e propria svolta nell'approccio diagnostico alla malattia e dunque anche al suo trattamento.

\section{Il percorso diagnostico}

Il percorso diagnostico si compone di tre momenti ben distinti: il sospetto clinico (quando pensarci), la conferma diagnostica mediante biopsia tissutale e l'identificazione del tipo di amiloidosi (tipizzazione).

\section{Quando pensarci}

Per fare diagnosi, occorre per prima cosa sospettare le presenza della malattia attraverso quelli che possiamo definire i suoi più caratteristici quadri clinici. È vero che di fatto la malattia può interessare tutti gli organi e apparati, tuttavia esistono alcuni quadri clinici particolarmente suggestivi anche se non specifici perché condivisi da altre più comuni patologie. Ecco perché l'amiloidosi è stata anche chiamata la grande "simulatrice" (1). 

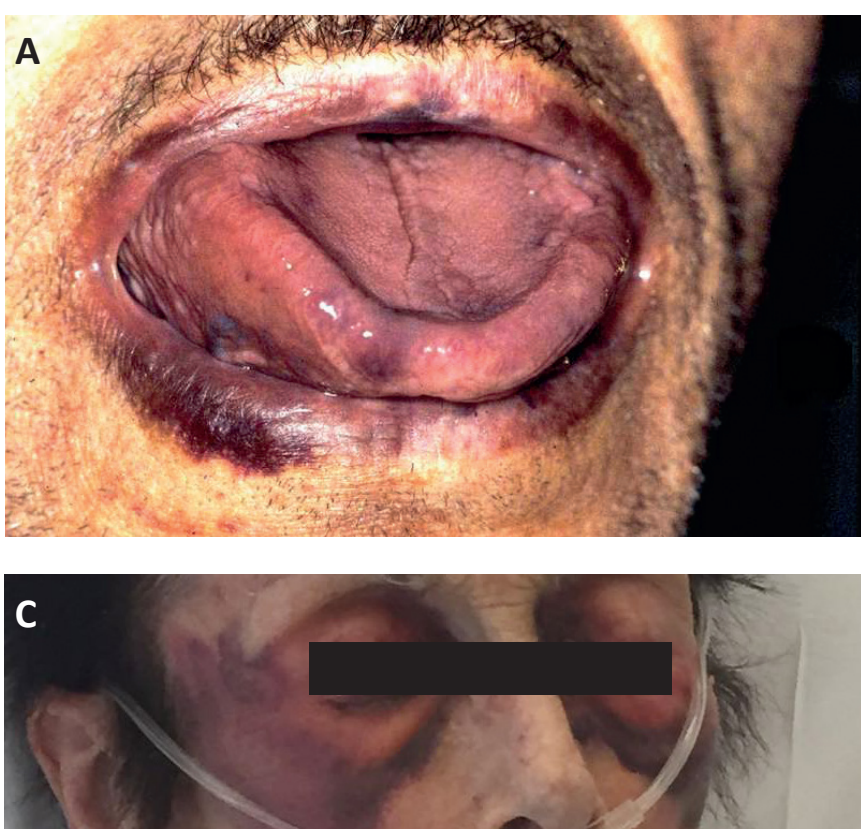

I quadri clinici più comuni delle amiloidosi sistemiche $(A L$, AA, ATTRm e ATTRwt) sono i seguenti:

Proteinuria/Sindrome nefrosica (con o senza insufficienza renale). II rene è assieme al cuore l'organo più frequentemente colpito dalla malattia anche se i segni del suo coinvolgimento (proteinuria e/o insufficienza renale) purtroppo non sono specifici. La presenza di una proteinuria più o meno importante con o senza insufficienza renale (IR) e magari con reni di dimensioni aumentate può essere suggestiva di amiloidosi in una popolazione adulto-anziana soprattutto in presenza di alcuni segni bioumorali, considerati vere e proprie "spie" della malattia.

Dispnea e scompenso cardiaco, se si accompagnano a un aumento degli spessori del setto interventricolare (IV) e della parete posteriore (PP) in presenza di segni di disfunzione diastolica e magari di un piccolo versamento pericardico possono essere segni del coinvolgimento cardiaco soprattutto se in presenza delle "spie" bioumorali della malattia.

Un'epatopatia colostatica con ittero o iperbilirubinemia, incremento delle gamma-GT e della fosfatasi alcalina sono i segni più caratteristici del coinvolgimento epatico.

La diarrea è il sintomo tipico dell'interessamento gastroenterico e dipende dal coinvolgimento locale del sistema nervoso vegetativo ma comuni possono essere anche i segni di pseudo ostruzione/ostruzione, di sanguinamento, la disfagia e il vomito a seconda del livello anatomico interessato dalla malattia.

Una polineuropatia sensitivo-motoria a partenza dagli arti inferiori e con andamento centripeto può essere il segno di un interessamento del sistema nervoso periferico. Comuni dell'interessamento del sistema nervoso vegetativo (SNV) sono anche altri sintomi tra cui l'ipotensione ortostatica, l'impotenza ecc.

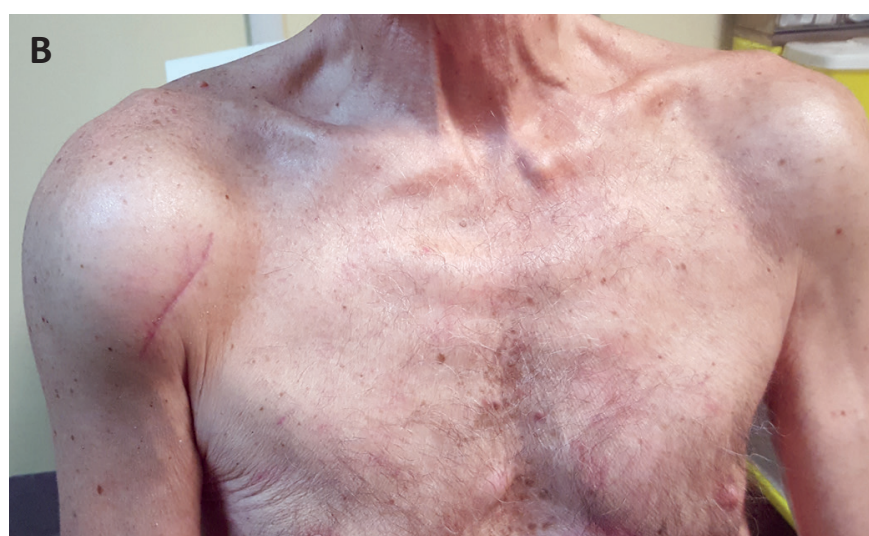

Fig. 1 - Amiloidosi, Segni Caratteristici (A) Macroglossia; (B) Spalle "imbottite"; (C) Ecchimosi periorbitarie.

Infine ci sono segni molto caratteristici e altamente suggestivi della malattia sebbene presenti solo in una minoranza dei pazienti: la macroglossia con più o meno evidenti impronte dentarie sul bordo della lingua, le ecchimosi periorbitarie e la cosiddetta "spalla imbottita" per infiltrazione dei tessuti periarticolari (Fig. 1).

Altri segni/sintomi molto frequenti e comuni a quasi tutte le forme di amiloidosi sono quelli relativi alla sindrome del tunnel carpale, sindrome che può precedere anche di molti anni le altre manifestazioni cliniche della malattia e la stessa diagnosi. Comune a molte forme di amiloidosi è la presenza di astenia e perdita di peso.

Tutti questi quadri clinici, di per sé non specifici o esclusivi dell'amiloidosi, acquistano tuttavia un significato molto suggestivo e ci devono spingere a ricercarne la presenza se si verificano all'interno di un contesto bioumorale particolare, vera e propria "spia" della malattia, come può essere quello legato alla presenza di una componente monoclonale (CM) nel siero e/o nelle urine (ad esempio, in corso di MGUS/Mieloma), di una malattia infiammatoria cronica con elevati indici di flogosi (come un aumento della siero amiloide A o SAA) oppure nell'ambito di una storia di familiarità per cardiopatia e/o polineuropatia. Questi particolari contesti clinico-bioumorali sono infatti la condizione necessaria anche se non sufficiente per lo sviluppo di una amiloidosi: AL nel primo caso, AA o reattiva nel secondo caso o ereditaria nell'ultimo (2).

Perciò tutte le volte che troviamo un danno d'organo suggestivo nell'ambito di uno di questi contesti clinico-bioumorali dobbiamo procedere nella fase successiva del percorso diagnostico, quella della verifica del nostro sospetto median- 
te la biopsia tissutale.

Va altresì sottolineato come questi contesti bioumorali vadano sempre scrupolosamente ricercati con una attenta anamnesi e richiedendo gli opportuni esami laboratoristici.

Questi, nel caso si voglia indagare la presenza di una CM ovvero di un clone plasmacellulare devono sempre includere una immunofissazione del siero e delle urine (Bence Jones qualitativa e quantitativa), un dosaggio sierico delle catene leggere libere* (3) e un dosaggio delle immunoglobuline. Questa procedura se usata in modo sistematico e corretto consente di identificare fino al $99 \%$ delle CM.

In questa sede va poi ricordato che il monitoraggio/followup dei pazienti con MGUS/Mieloma smoldering deve sempre includere la ricerca di proteinuria e il dosaggio sierico di NTproBNP e Troponina I o T come spie sensibili di un precoce coinvolgimento renale o cardiaco da amiloidosi.

\section{La biopsia tissutale}

Rappresenta un atto dovuto nel percorso diagnostico di ogni paziente affetto da amiloidosi e resta lo strumento fondamentale per confermare il sospetto clinico e stabilire senza ombra di dubbio la presenza della malattia. Senza la dimostrazione della presenza dei depositi di amiloide all'interno di organi o tessuti non possiamo fare diagnosi "certa" di amiloidosi. La diagnosi istologica si basa sul reperto di materiale rosso Congo positivo che assume la caratteristica birifrangenza "verde mela" quando osservato in luce polarizzata.

Fino alla fine degli anni Ottanta del Novecento il ricorso alla biopsia degli organi sospettati avveniva sporadicamente o tardivamente e spesso il materiale raccolto (biopsia gengivale, rettale ecc.) non era rappresentativo per cui il più delle volte la diagnosi finiva per essere autoptica. Alla fine degli anni Ottanta veniva con successo proposta da Merlini et al a Pavia una metodica semplice, non invasiva, facilmente ripetibile e altamente sensibile e specifica che consentiva, ancorché in mani esperte, di fare rapidamente la diagnosi: la metodica dell'aspirato del "grasso periombelicale" (GPO) già proposta nel 1973 dallo svedese Peer Westermark (4).

\section{II prelievo del grasso periombelicale (GPO)}

È l'indagine di elezione e può essere eseguita in tutti i pazienti con l'eccezione di quelli che presentano un'ernia ombelicale. La metodica consiste nell'aspirare piccoli frammenti di tessuto adiposo dalla regione periombelicale e di schiacciarli tra due vetrini per poi separare i due vetrini e colorarli con rosso Congo (2). La sua sensibilità è variabile, oscillando a seconda delle casistiche e del tipo di amiloidosi tra l'80\% e

\footnotetext{
* Oggi esistono in commercio diversi kit disponibili, di cui tuttavia l'unico ampiamente documentato e validato è quello di Binding Site (UK).
}
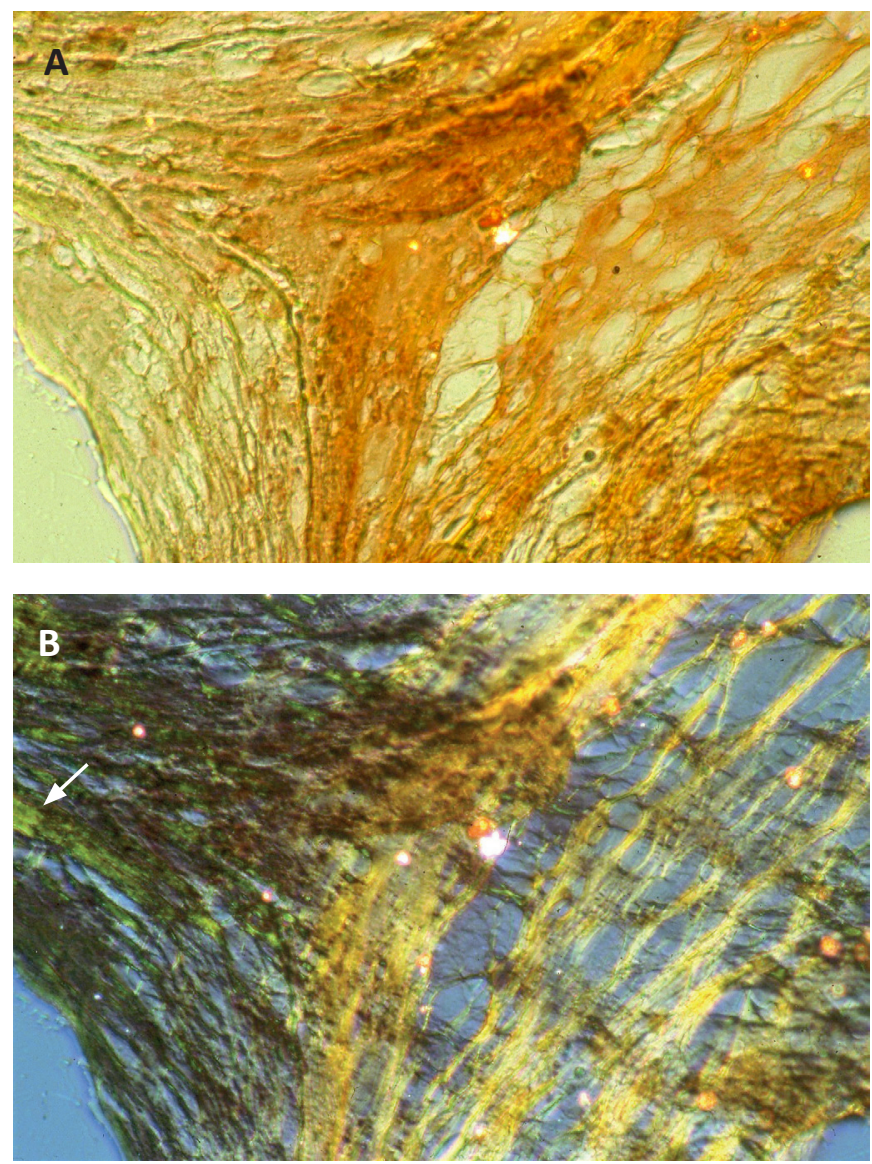

Fig. 2 - Agoaspirato di Grasso Periombelicale Osservazione in Microscopia Ottica (×200) dopo Colorazione con Rosso Congo (A) e osservazione in luce polarizzata (B), positiva per la presenza di depositi di amiloide.

il $90 \%$ nelle AL e nelle ATTRmutate e tra il $70 \%$ e l' $80 \%$ nelle forme AA. La sua specificità è del 94\% circa (1) (Fig. 2). Unico vero limite della metodica è rappresentato dalla necessità di acquisire una sufficiente esperienza per la lettura del preparato, non sempre disponibile nei Centri periferici.

Nel caso di un risultato negativo si può procedere a effettuare la biopsia delle ghiandole salivari minori, metodica altrettanto semplice e affidabile con una sensibilità del $60 \%$ circa nei casi GPO negativi. Solo se anche questa risulta negativa e resta elevato il sospetto clinico di malattia, possiamo e dobbiamo procedere alla biopsia dell'organo interessato, premunendoci tuttavia di ridurre al minimo il rischio di sanguinamento. Questa eventualità, ritenuta spesso una importante limitazione alla biopsia d'organo, si è rivelata in effetti meno frequente del previsto purché vengano prese tutte le precauzioni del caso, tra cui il controllo della concentrazione plasmatica del fattore $X$, talora ridotto a causa del suo intrappolamento aspecifico nei depositi di amiloide (2).

Il risultato positivo del GPO ci consente non solo di porre la diagnosi di amiloidosi ma anche di considerare quest'ultima 


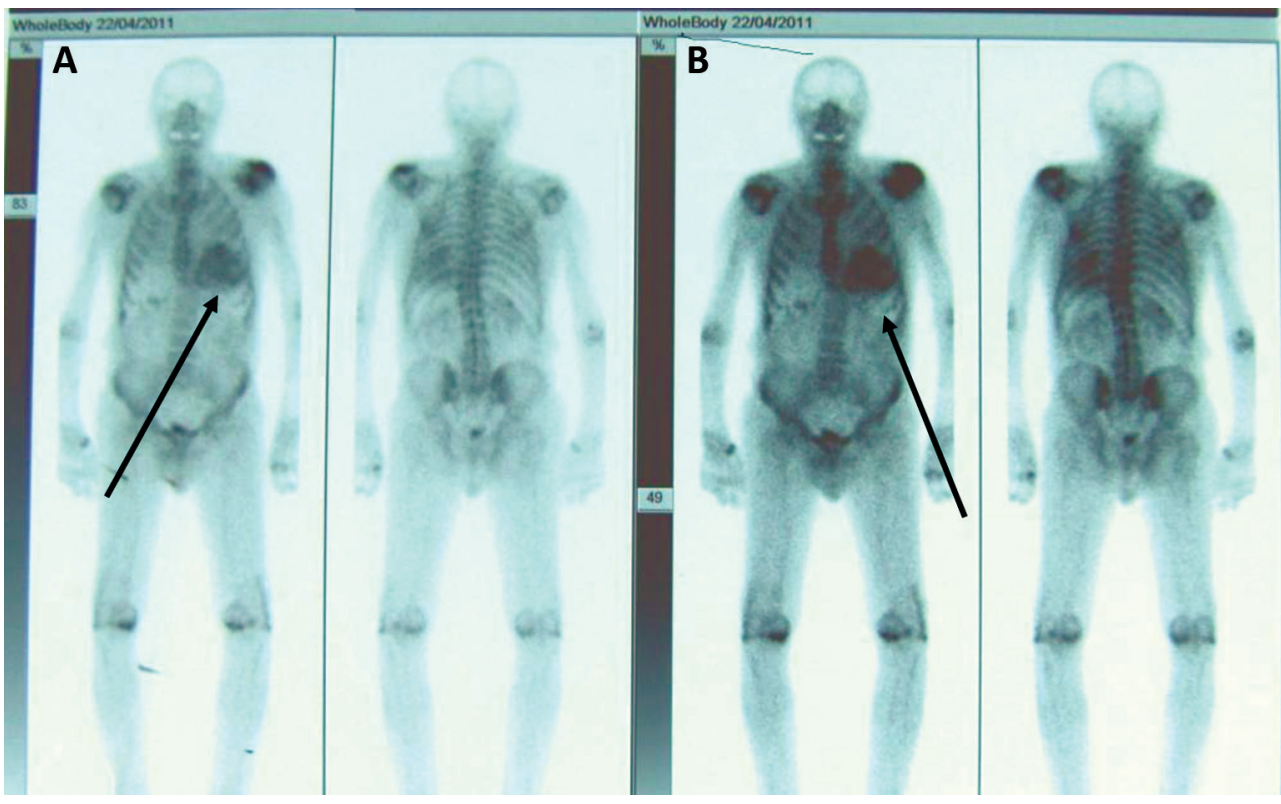

Fig. 3 - Amiloidosi cardiaca Senile Scintigrafia Ossea con 99Tc Difosfonati: netta captazione del tracciante a livello cardiaco. come la causa più probabile del danno d'organo presentato dal paziente (sindrome nefrosica, scompenso cardiaco ecc.). Ovviamente dobbiamo escludere l'esistenza di altre cause potenzialmente responsabili di quel danno d'organo.

\section{Biopsia dell'organo interessato (biopsia renale, cardiaca ecc.)}

La biopsia dell'organo interessato, una volta verificate le capacità emostatiche del paziente, può e deve essere praticata tutte le volte che persistono ragionevoli dubbi sulla natura del processo morboso che coinvolge l'organo. Essa sola infatti ci garantisce in questi casi dell'appropriatezza della diagnosi.

Inoltre, nel caso specifico della biopsia renale (ma l'esempio potrebbe essere estensibile anche ad altri organi) essa sola consente di identificare altre forme di coinvolgimento renale da amiloidosi, altrimenti non identificabili (vedi Amiloidosi renali).

\section{Una eccezione che conferma la regola: I'amiloidosi senile}

La sensibilità del GPO è praticamente minima nelle forme di amiloidosi senile da TTRwt e si aggira nella nostra esperienza attorno al $15 \%$ circa dei casi (dati personali). Dal momento che questa forma di amiloidosi presenta un tipico e pressoché esclusivo coinvolgimento cardiaco, l'unico modo per arrivare alla diagnosi era, fino a pochi anni fa, quello di eseguire una biopsia endomiocardica, difficilmente eseguibile tuttavia data l'età avanzata e le spesso critiche condizioni cliniche dei pazienti. Questa importante limitazione ha di fatto impedito la diagnosi di amiloidosi senile in molti pazienti anziani con interessamento cardiaco fino a quando non è stato osservato che il tessuto miocardico di questi pazienti mostrava una forte e atipica captazione dei traccianti contenenti difosfonati normalmente utilizzati per le scintigrafie ossee. Tale captazione è specifica dei pazienti affetti da amiloidosi da TTR sia mutata (TTRm) che wild type (TTRwt) (Fig. 3).

In questi casi la ricerca di mutazioni del DNA permette di differenziare le forme mutate da quelle wt di amiloidosi cardiaca da TTR purché non vi sia la contemporanea presenza di una $\mathrm{CM}$, condizione che non consente di poter escludere con certezza una forma $A L$, poiché anche in queste forme si può talora osservare una captazione cardiaca del tracciante. In questi casi è inevitabile il ricorso alla biopsia endomiocardica. Nei casi dove la CM è assente è stata ammessa la possibilità di fare diagnosi di ATTR mutata o wild type anche in assenza di una biopsia tissutale positiva (5) (Fig. 4).

\section{La tipizzazione}

Una volta posta la diagnosi di amiloidosi, dobbiamo capire di che tipo di amiloidosi si tratta poiché da questo dipende il trattamento da seguire. Quest'ultimo passaggio è quello più critico dell'intero percorso diagnostico e non è scontato.

Se infatti il contesto clinico e bioumorale del paziente è di solito suggestivo del tipo di amiloidosi che abbiamo di fronte ( $A L$ se il paziente è portatore di una $C M, A A$ se è affetto da una malattia reumatica cronica con elevati indici di flogosi o ATTR se abbiamo il cuore da solo o in associazione a una polineuropatia) questo tuttavia non deve trarci in inganno e indurci a conclusioni affrettate.

Infatti è sempre più frequente il riscontro di una CM, soprattutto nella popolazione adulto-anziana, la cui presenza è spesso casuale e non risulta responsabile del quadro clinico. La presenza 


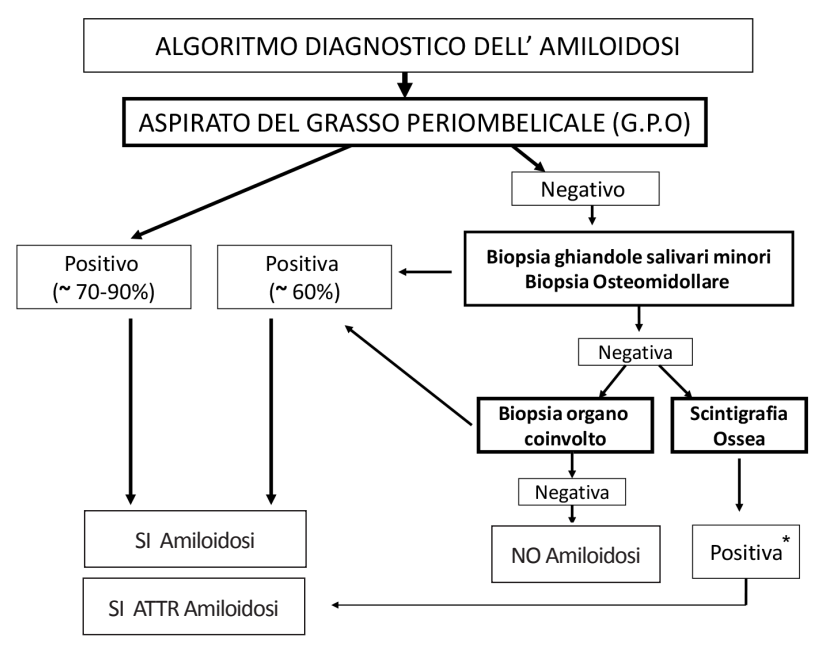

Fig. 4 - Algoritmo Diagnostico. * La diagnosi di Amiloidosi da TTR è valida solo in assenza di una $\mathrm{CM}$ e con una Scintigrafia da moderatamente a fortemente positiva $\left(2^{\circ}-3^{\circ}\right.$ grado).

di una $\mathrm{CM}$ viene oggi riferita attorno a un 8-10\% della popolazione di età $>70$ anni aumentando ulteriormente col crescere dell'età. Nei pazienti affetti da amiloidosi cardiaca senile il riscontro di una CM è stato segnalato addirittura nel $15-20 \%$ dei casi (XV Congresso Internazionale sull'Amiloidosi, Uppsala (Svezia) 2016). Purtroppo in passato sono stati fatti grossolani errori diagnostici per aver identificato automaticamente come AL tutte le forme cliniche che presentavano una $\mathrm{CM}$ con conseguenze terapeutiche devastanti per i pazienti (6). Se infatti la presenza di una $\mathrm{CM}$ è necessaria per porre diagnosi di AL, non è tuttavia sufficiente per confermarla con certezza. Nell'insieme, il rischio di un errore nella definizione della natura della malattia non è trascurabile, poiché l'associazione di due potenziali cause di amiloidosi concomitanti nello stesso paziente (ad esempio, la presenza di una componente monoclonale in un paziente con una mutazione genetica della transtiretina o apolipoproteina $\mathrm{A}-\mathrm{I}$, o con una patologia infiammatoria cronica) non è un evento infrequente.

La definizione certa del tipo di amiloidosi richiede l'esame del preparato in immunoistochimica mediante l'impiego di anticorpi diretti contro le diverse proteine amiloidogeniche: catene leggere $k$ o lambda delle Ig per la conferma di $A L$, siero amiloide $A(S A A)$ per le forme reattive (AA), oppure la molecola della transtiretina per le forme da TTR. Purtroppo l'immunoistochimica tradizionale in microscopia ottica (come anche l'immunofluorescenza su preparati renali), a causa della scarsa sensibilità e specificità degli anticorpi rivolti verso le catene leggere $k$ e lambda, non permette una tipizzazione inequivocabile dei depositi nell'amiloidosi AL. Diversamente, avendo gli anticorpi anti-SAA e anti-TTR una buona specificità, è possibile formulare con sufficiente precisione una diagnosi di amiloidosi reattiva (AA) o da TTR se in presenza di una mutazione nel gene di questa molecola

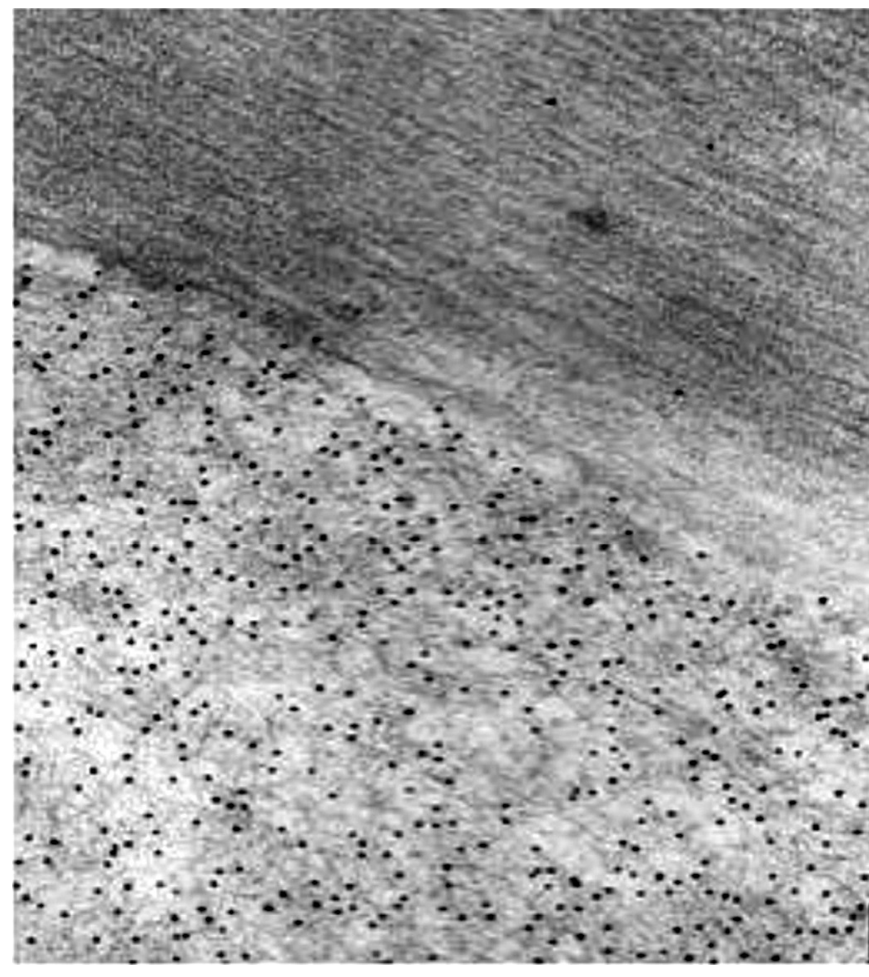

Fig. 5 - Immunogold Caratterizzazione immunoistochimica in ME di fibrille di amiloide con anticorpi anti catene leggere Lambda coniugati a particelle di oro colloidale (puntini neri).

$(1,2)$. L'analisi genetica della molecola della TTR deve sempre completare il processo della tipizzazione, soprattutto nelle forme da TTR.

In tutti gli altri casi è indicata la caratterizzazione ultrastrutturale delle fibrille di amiloide mediante l'uso di anticorpi marcati con particelle d'oro (tecnica dell'immunogold o immunomicroscopia elettronica) che permette la co-localizzazione degli anticorpi con le fibrille specifiche (Fig. 5). In casi particolarmente difficili, le tecniche di proteomica mediante microdissezione laser e spettroscopia di massa dei preparati istologici che contengono i depositi di amiloide consentono di identificare in modo inequivocabile la proteina che forma $i$ depositi di amiloide $(2,7)$.

In nessun caso è giustificato iniziare la terapia in assenza di una tipizzazione certa dei depositi di amiloide.

\section{Amiloidosi renali}

Si conoscono oggi numerose forme di coinvolgimento renale in corso di amiloidosi, alcune molto diverse tra loro e dallo stereotipo tradizionale dell'amiloidosi renale rappresentato da una proteinuria nefrosica con o senza insufficienza renale. Alcune di esse sono state identificate molto recentemente grazie all'impiego della proteomica applicata alla biopsia renale (6). In conclusione possiamo riassumere le diverse forme di coinvolgimento renale in corso di amiloidosi in due principali categorie: 
TABELLA I - Le diverse forme di Amiloidosi Renali oggi conosciute e le loro principali caratteristiche istopatologiche e cliniche

\begin{tabular}{lllll}
\hline Tipo di amiloidosi & Origine & $\begin{array}{l}\text { Localizzazione } \\
\text { Corticale/Midollare }\end{array}$ & Segni/Sintomi Renali & Altri Organi Interessati \\
\hline AL & acquisita & Corticale & Proteinuria \pm IR & Cuore, fegato, SNP, GI... \\
AA & acquisita & Corticale & Proteinuria \pm IR & Fegato, cuore, milza T Gastro-int. le \\
ATTR & ereditaria & Corticale & proteinuria & SNP, Cuore \\
ALECT2 & acquisita & Corticale (Interstizio) & Proteinuria \pm IR & Fegato, milza, polmoni \\
AFibrinogeno & ereditaria & Corticale (glomeruli) & Proteinuria $\pm I R$ & Non noto \\
ALisozima & ereditaria & Corticale & Proteinuria $\pm I R$ & Tratto Gastrolntestinale, cute \\
AApoAI & ereditaria & Midollare & IRC & Cuore, fegato, cute, SNP \\
AApoAII & ereditaria & Corticale & Proteinuria & Non noto \\
AApoAIV & acquisita & Midollare & IRC & Non noto \\
AH & acquisita & Corticale & Proteinuria, microematuria,IRC & Cuore \pm \\
AHL & acquisita & Corticale & Proteinuria, microematuria,IRC & Cuore \pm \\
AApoCII & ereditaria & Corticale & Proteinuria $\pm I R$ & Non noto \\
AApoCIII & ereditaria & Corticale & Proteinuria $\pm I R$ & Non noto \\
AGelsolina & ereditaria & Corticale & Proteinuria, IRC progressiva & SNP, Nervi cranici
\end{tabular}

le forme corticali, di gran lunga le più frequenti, in cui i depositi di amiloide si localizzano all'interno delle diverse componenti del tessuto corticale e le forme midollari nelle quali i depositi sono confinati esclusivamente all'interno della midollare.

Nel primo gruppo troviamo le forme $A L, A A$ e altre che interessano prevalentemente $\mathrm{i}$ glomeruli ma anche $\mathrm{i}$ vasi e $\mathrm{i}$ tubuli e altre meno conosciute come la ALECT2 con interessamento pressoché esclusivo del solo interstizio corticale e quelle da fibrinogeno alfa che, contrariamente, interessano massicciamente e quasi esclusivamente i glomeruli (Tab. I).

Tra le seconde conosciamo al momento solo due forme: AApoAl e AApoAIV. La prima è una forma legata a una mutazione della molecola di ApoAl del C-HDL e quindi è una forma ereditaria che interessa rene e cuore. La seconda non sembra una forma legata a mutazioni della molecola di ApoAIV.

Il coinvolgimento renale in entrambe è atipico e si esprime con la presenza di insufficienza renale di lieve-moderata entità che può evolvere verso l'uremia terminale come unico segno del coinvolgimento dell'organo.

La scoperta di queste nuove forme di amiloidosi renali sottolinea l'importanza della biopsia renale abbinata all'uso sistematico della colorazione con rosso Congo come strumento diagnostico fondamentale per poter fare diagnosi di amiloidosi in tutti quei casi paucisintomatici e a evoluzione insidiosa di insufficienza renale cronica. L'uso della proteomica consente poi di identificare la proteina amiloidogenica che ne sta alla base.

\section{Disclosures}

Financial support: No financial support was received for this submission. Conflict of interest: The authors have no conflict of interest.

\section{Bibliografia}

1. Amyloidosis: diagnosis and treatment. Gertz MA and Rajkumar SV eds. Springer, Berlin, Germany: 2010.

2. www.amiloidosifirenze.it

3. Bradwell AR, Carr-Smith HD, Mead GP, et al. Highly sensitive, automated immunoassay for immunoglobulin free light chains in serum and urine. Clin Chem. 2001; 47(4):673-80.

4. Westermark P, Stenkvist B. A new method for the diagnosis of systemic amyloidosis. Arch Intern Med. 1973;132(4):522-3.

5. Gillmore JD, Maurer MS, Falk RH, et al. Nonbiopsy diagnosis of cardiac transthyrethin amyloidosis. Circulation. 2016;133 (24):2404-12.

6. Lachmann HJ, Booth DR, Booth SE, et al. Misdiagnosis of hereditary amyloidosis as AL (primary) amyloidosis. N Engl J Med. 2002;346(23):1786-91.

7. Obici L, Nuvolone M, Merlini G. Expanding the spectrum of systemic amyloid diseases: a new hint from the kidney. Kidney Int 2016; 90:479-481 